\section{Attaching Spheres to Cantilevers for Colloidal Probe Force Measurements: A Simplified Technique}

\author{
Yang Gan \\ University of Newcastle, Callaghan, NSW, Australia \\ yang.gan@newcastle.edu.au
}

\section{Introduction}

The usage protocol of the Atomic Force Microscope (AFM) known as the colloidal probe technique [1] enables researchers to investigate quantitatively the surface and interface forces in a far greater number of materials. The innovative essence of the colloidal probe technique was to modifying an AFM probe cantilever by attaching a micron sized particle to it. Although any shaped particle can be attached to the cantilever, a sphere is preferred. The most widely employed sphere attaching technique uses a 3-D manipulator to move one sharpened metal wire to pick up a tiny glue droplet and deposit it on the foremost part of the cantilever of the probe. Then another wire is used to pick up a sphere and attach it to the cantilever via the glue droplet. The above procedure is referred to as the "two wires" technique.

But in practice, the writer found that the above procedure has several disadvantages. First, it's time consuming, especially the picking-up-a-sphere step. It is believed that the capillary force between the wire Fig. 1 and sphere give the wire "picking-up power." But, more often than not, one must try

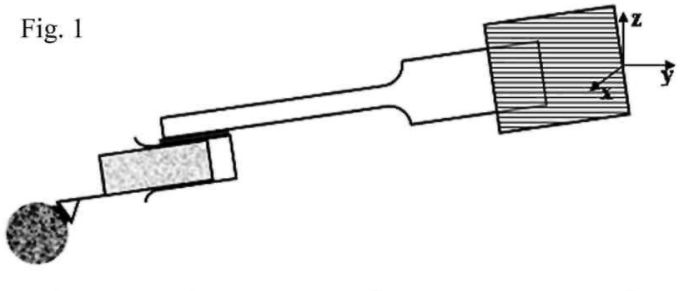
many times to pick up a sphere--even for an experienced experimentalist. Second, the preparation of suitable sharpened wires is time consuming. Lastly, another serious disadvantage of the two-wire method is that it is not easy to discover if the cantilever is broken, this is very frustrating in that the researcher will only discover that the cantilever is defective later when beginning force measurements. It is simple to replace the cantilever when it is operated in air, but it's annoying to replace it when it is operated in a liquid solution: one has to take apart, clean and reassemble every part of the liquid cell.

In the literature, there have been descriptions of alternative techniques without using wires: Huntington and Nespolo [2] described in detail steps for gluing a sphere to a cantilever aided by the unique advantage of Dimension ${ }^{\mathrm{TM}} 3100$ AFM; Raiteri, et al. [3] described how to glue a sphere to a tipless cantilever under a light microscope by a moving cantilever, but the steps are not detailed. In this note, a technique similar to that of Raiteri, et al. is presented in detail.

\section{Experimental procedures and discussion}

The essence of this technique is that instead of sharpened wires, a probe is moved with a 3-D manipulator to pick up

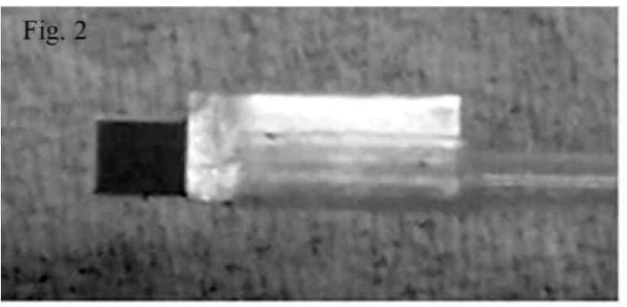
glue and capture a

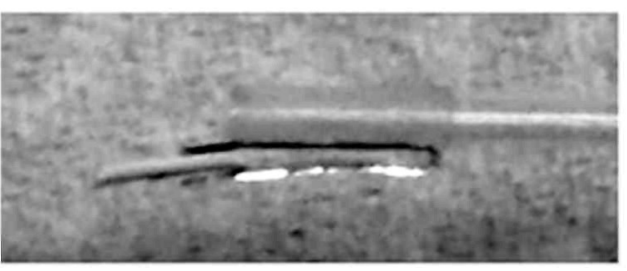
sphere and then attach the sphere to the cantilever. The steps are as follows:

(1)Bend a piece of aluminium foil (size $10 \times 2 \times 0.1 \mathrm{~mm}$ ) in the middle along the narrow side into a U-shaped clip. Adjust the distance between opposite sides of the clip to allow a silicon nitride probe to be comfortably inserted into and firmly held by the clip. Take a clean, glass, pipette tube and wrap double-sided tape for about $5 \mathrm{~mm}$ at the narrow end of the tube. Firmly attach the U-shaped clip to the tape along the tube. In this way, a probe holder is fabricated (see Fig. 1 for a schematic). Note that any long rigid rod that is suitable can be inserted into the arm of the manipulator, but we find that a pipette tube is a handy choice.

(2)Insert a probe into the clip with the tips facing downward; allow about half of the probe to extend outside the clip (see Fig. 2 for the top and side view).

(3)Fix the other end of the pipette tube into the arm of a 3-D micromanipulator, and make sure that the tube is tilted with respect to the horizontal surface (see Fig. 1); also check visually that the narrow edge of the probe is parallel to the glass slide below.

(4)Spread spheres on a clean glass slide, and put a small drop of glue nearby on the slide.

(5)While monitoring through an optical microscope, position the probe so that it is close to the edge of the glue drop, lower the probe to press gently against the glass slide, push the probe into the glue (note that only the foremost tip of the cantilever needs to touch the glue), then translate out for several microns across the slide surface so that excess glue is dragged across the surface
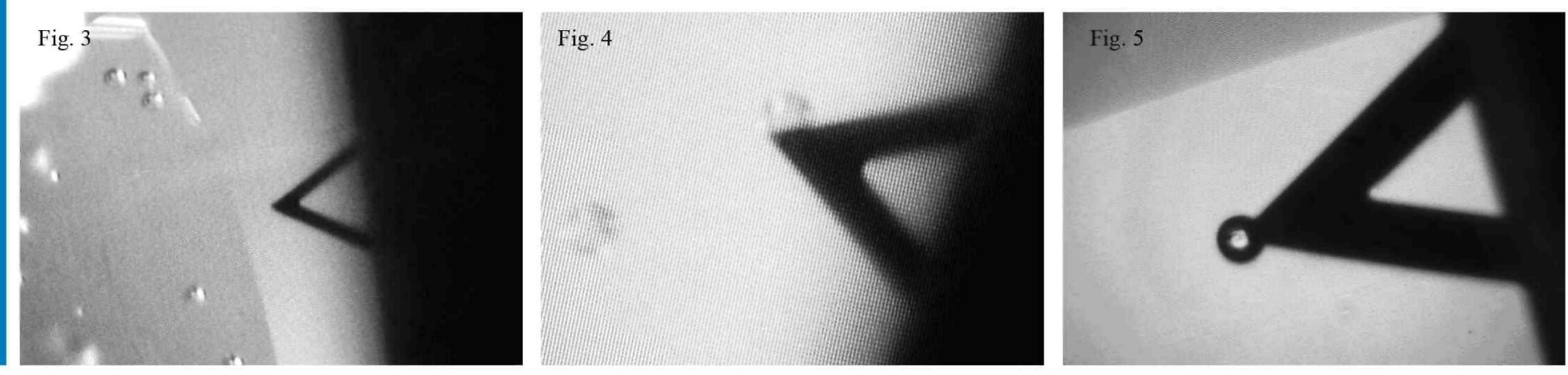


\section{It's Difficult To Do A}

Side-By-Side Comparison

\section{When Your Coater}

\section{Stands Alone.}

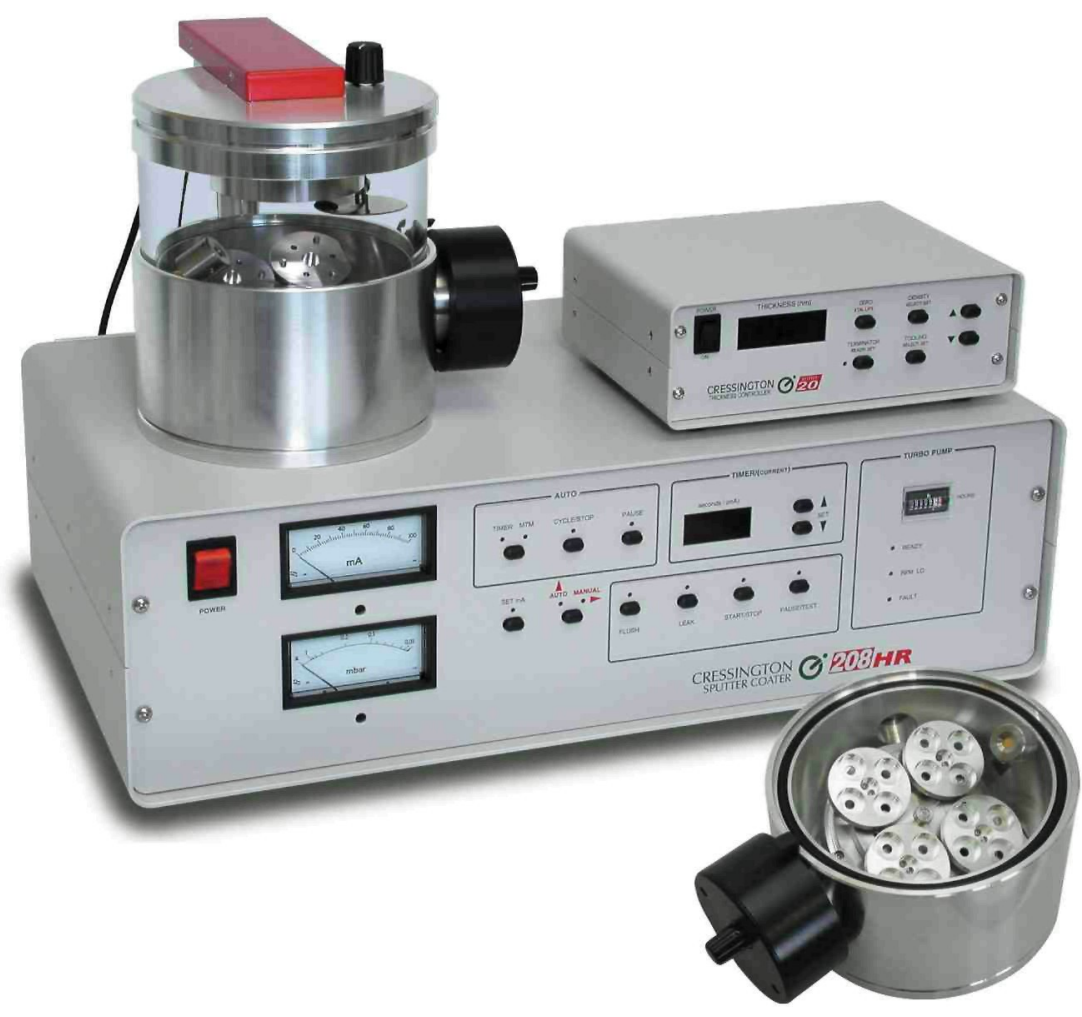

\section{High Resolution Sputter Coater 208HR for FE-SEM}

Superior Features:

- Wide Choice of Coating Materials

- Precision Thickness Control

- Multiple Sample Stage Movements

- Variable Chamber Geometry

- Wide Range of Operating Pressures

- Compact Modern Benchtop Design
D TED PELLA, INC. Microscopy Products for Science and Industry
4595 Mountain Lakes Blvd., Redding, CA 96003-1448

Phone: $530-243-2200$ or 800-237-3526 (USA) • FAX: 530-243-3761

Email: sales@tedpella.com 
with the end result that only a very small amount of glue remains on the tip of the cantilever (see Fig. 3). One advantage of pressing the cantilever against the surface is that one can easily check to see if the cantilever is good or broken. Replace the cantilever if it is broken.

(6)Lift the probe and move it to locate spheres on the same glass slide, position the tip of the cantilever on the top of a sphere (see Fig. 4), lower vertically and allow the cantilever's tip to touch a sphere and then quickly lift up. If positioned well, the sphere is attached to the foreside of the pyramid-shaped tip (see Fig. 1 and Fig. 5). Note that when a sphere fails to be glued to the cantilever, one should leave this sphere and try another one, since the just-touched sphere may roll along the glass slide and very probably get coated with glue if it is touched repeatedly with the cantilever.

(7)Release the probe from the clip and keep it in a clean place for the glue to cure before beginning force measurements.

Our experience established that this technique is much more efficient than the routine "two wires" method we can produce five colloidal probes tips in about 20 minutes.

\section{Acknowledgment}

The author would like to acknowledge support from the Australian Research Council for Discovery Grant DP0343326 and the University of Newcastle for support through the University of Newcastle Research Fellow Scheme. Thanks to Dr. Erica Wanless for the use of the AFM facility; Dr. George Franks for discussing and proofreading the paper and to Glen McCann for stimulating discussions and testing the new technique.

\section{References}

1. W. A. Ducker, T. J. Senden, R. M. Pashley, Measurement of Forces in Liquids Using a Force Microscope, Langmuir 8, 1831 (1992).

2. S. Huntington, S. Nespolo, Precision Attachment of (Silica) Spheres to AFM Cantilevers Tips, Microscopy Today 9, 32 (2001).

3. R. Raiteri, M. Preuss, M. Grattarola, H.-J. Butt, Preliminary Results On The Electrostatic Double-layer Force Between Two Surfaces With High Surface Potentials, Colloid Surf. A 136, 191 (1998).

\section{Nissl: The Man, The Stain and The Substance}

\author{
John A. Kiernan \\ Department of Anatomy \& Cell Biology \\ The University of Western Ontario London, Canada \\ jkiernan@uwo.ca
}

Nissl staining of nervous tissue is widely practised to show the distributions of layers and groups (nuclei) of neurons. Commonly, the staining is carried out to provide an anatomical framework for immunohistochemical and electrophysiological studies. Since the early days of histochemistry and electron microscopy, it has been known that the basophilic granules or stripes in the cytoplasm of a neuronal cell body are composed of granular endoplamic reticulum, with the staining properties being attributable to ribosomal RNA. How did these stainable organelles come to be called the Nissl substance?

Franz Nissl (1860-1919) was a German neuropsychiatrist, a physician who took on the whole gamut of diseases affecting the nervous system and also carried out histopathological studies of his deceased patients. He developed his staining technique in 1884, while he was a medical student in Munich. He wrote an essay on the pathology of the cells of the cerebral cortex, entered it in a competition, and won the prize. The formal publication of the staining method and description of the revealed intracellular details appeared ten years later as a series of papers in Volume 13 of the journal Neurologisches Centralblatt.

After obtaining his doctorate in 1885, Nissl became an assistant to Johan Bernhard Aloys von Gudden (1824-1886), a prominent neuropsychiatrist now remembered especially for his contributions to neuroanatomy. In 1886, von Gudden was probably murdered by an ungrateful patient, King Ludwig II of Bavaria. von Gudden and two other distinguished neuropsychiatrists had recently examined Ludwig, who had brought his country close to bankruptcy by building fairytale castles and palaces. They made a diagnosis that we would now call paranoid schizophrenia. The king was relieved of all official responsibilities and taken by von Gudden to Schloss Berg. On the evening of June 12th 1886, the two men went for an evening walk by the nearby Lake Starnberg. Their bodies were found at the water's edge later that night. It is widely believed that the mad king killed his physician and then committed suicide by drowning, but it is also possible that both men were murdered as part of a conspiracy (Kleinert, 2001).

In the decade following, now in Frankfurt, Nissl worked alongside Ludwig Edinger (1855-1919), Carl Weigert (1845-1904) and Alois Alzheimer (1864-1915), whose names have long been household words in the world of neuroscience. In 1895 Nissl moved to the University of Heidelberg, invited by the great psychiatrist Emil Kraepelin, whom he succeeded as professor in 1901. In 1918 Kraepelin moved back to Munich and collaborated for a year with Korbinian Brodmann (1868-1918), whose numbering system for cortical areas, based on the structure revealed in Nissl-stained sections, is in widespread use to this day.

Nissl called the basophilic objects in neurons granules; the terms Nissl granules, Nissl bodies, Nissl substance and tigroid bodies are all synonymous. They stain with cationic dyes - Nissl used methylene blue - on account of their high content of RNA. The first high quality electron micrographs of neurons (Dawson et al. 1955; Palay \& Palade, 1955) revealed that the Nissl substance was rough endoplasmic reticulum: parallel membranes associated with granules that were later called ribosomes. George Emil Palade (born 1912) shared the Nobel Prize in Physiology or Medicine in 1974 for describing the structures and functions of organelles in cells. The other recipients were Albert Claude (1898-1983) for isolation and biochemical study of organelles, and Christian Rene de Duve (born 1917) for the discovery of lysosomes.

\section{References}

Dawson IM, Hossack J, Wyburn GM. 1955. Observations on the nissl's substance, cytoplasmic filaments and the nuclear membrane of spinal ganglion cells. Proc. Roy. Soc. London B 144:496-506.

Kleinert R. 2001. Franz Nissl. http://www.whonamedit.com/doctor.cfm/2465. html

Palay SL, Palade GE 1955. The fine structure of neurons. J. Biophys. Biochem. Cytol. 1:69-88. 